\title{
28 Research Square \\ Radiomics Nomogram Based on Spectral CT Imaging to Diagnose Osteoporosis
}

\section{Qianqian Yao}

The Second Affiliated Hospital of Shandong First Medical University

\section{Mengke Liu}

The Second Affiliated Hospital of Shandong First Medical University

\section{Kemei Yuan}

The Second Affiliated Hospital of Shandong First Medical University

\section{Yue Xin}

The Second Affiliated Hospital of Shandong First Medical University

\section{Xiaoqian Qiu}

The Second Affiliated Hospital of Shandong First Medical University

\section{Xiuzhu Zheng}

The Second Affiliated Hospital of Shandong First Medical University

\section{Changqin Li}

The Second Affiliated Hospital of Shandong First Medical University

\section{Shaofeng Duan}

GE Healthcare

jian qin ( $\square$ sdqinjian@126.com )

The Second Affiliated Hospital of Shandong First Medical University

\section{Research Article}

Keywords: Osteoporosis, Radiomics, Spectral CT

Posted Date: November 19th, 2021

DOI: https://doi.org/10.21203/rs.3.rs-1085520/v1

License: (9) (7) This work is licensed under a Creative Commons Attribution 4.0 International License. Read Full License 


\section{Abstract}

Background: Osteoporosis is associated with a decrease of bone mineralized component as well as a increase of bone marrow fat. At present, there are few studies using radiomics nomogram based fat-water material decomposition (MD) images of spectral CT as an evaluation method of osteoporosis. This study aims to establish and validate a radiomics nomogram based the fat-water imaging of spectral CT in diagnosing osteoporosis.

Methods: 95 patients who underwent spectral CT included T11-L2 and dual x-ray absorptiometry (DXA) were collected.

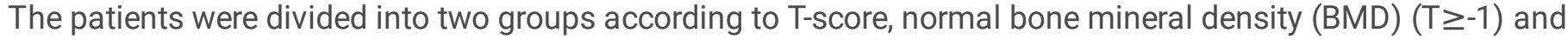
abnormally low BMD $(T<-1)$. Radiomic features were selected from fat-water imaging of the spectral $\mathrm{CT}$. Radscore was calculated by summing the selected features weighted by their coefficients. A nomogram combining the radiomics signature and significant clinical variables was built. The ROC curve was performed to evaluate the performance of the model. Finally, we used decision curve analysis (DCA) to evaluate the clinical usefulness of the model.

Results: Five radiomic features based on fat-water imaging of spectral CT were constructed to distinguish abnormally low BMD from normal BMD, and its differential performance was high with an area under the curve (AUC) of 0.95 (95\% $\mathrm{Cl}, 0.89-1.00)$ in the training cohort and $0.97(95 \% \mathrm{Cl}, 0.91-1.00)$ in the test cohort. The radiomics nomogram showed excellent differential ability with AUC of $0.96(95 \% \mathrm{Cl}, 0.91-1.00)$ in the training cohort and $0.98(95 \% \mathrm{Cl}, 0.93-1.00)$ in the test cohort, which performed better than the radiomics model and clinics model only. The DCA showed that the radiomics nomogram had a higher benefit in differentiating abnormally low BMD from normal BMD than the clinical model alone.

Conclusion: The radiomics nomogram incorporated radiomics features and clinical factor based the fat-water imaging of spectral CT may serve as an efficient tool to identify abnormally low BMD from normal BMD well.

\section{Introduction}

Osteoporosis is a systemic skeletal disease characterized by bone loss leading to an increased risk of fragility fractures [1]. With the aging of the population, the incidence of osteoporosis and fragility fractures is increasing. If not treated in time, these fractures would lead to high morbidity and mortality and carries social and economic burdens [2, 3 , 4]. Therefore, it is very important to early detect osteoporosis, and reduce the incidence of fracture complications.

At present, dual x-ray absorptiometry (DXA) is the most widely used screening tool for osteoporosis and preferred method for bone mineral density (BMD) measurement [5]. According to WHO guidelines [6], a DXA-derived T- score less than -1.0 indicates an abnormally low BMD, and when the T-score is greater than -1.0 is normal.

The recently introduced spectral CT, which is based on a single tube fast switching between low-energy (80 kV) and high-energy (140 kV) data sets, provides precisely material decomposition (MD) images (e.g. fat-water-based and iodine-water-based MD images) [7]. The spectral CT has been used clinically to quantitatively estimate calcium concentration in trabecular bone and cortical bone in patients under going hemodialysis with secondary hyperparathyroidism [8] and to differentiate small hepatic hemangioma from small hepatocellular carcinoma [9]. Osteoporosis is associated with a decrease of bone mineralized component as well as a increase of bone marrow fat, which is caused by a shift of differentiation of mesenchymal stem cells to adipocytes[10]. Fat-water-based MD images of spectral CT can reflect the changes of fat content in the process of osteoporosis. However, there are few studies using fat-water MD images of spectral CT as an evaluation method of osteoporosis.

The term radiomics has attracted increased attention in recent years, and it is a promising technique using computerized quantitative imaging analysis to extract an enormous quantity of image-related features, followed by 
subsequent data analysis for decision support [11-14]. Therefore, the purpose of this study is to establish and validate a radiomics-clinical model(radiomics nomogram) that combined the fat-water imaging of spectral CT based radiomics signature and clinical risk factors for discriminating abnormally low BMD from normal BMD.

\section{Materials And Methods 2.1 Patients}

This retrospective study was approved by the institutional review board, and the requirement to obtain informed consent was waved. The primary cohort of this study was identified by searching the institutional picture archiving and communication systems (PACS) database for medical records from January 2020 to August 2020 to identify patients with DXA as well as spectral CT examination for other indications in routine practice. For inclusion, patients had to have had thoracic or abdominal spectral CT that showed T11-L2 were included in the study, or both, as well as DXA of the hips and lumbar spine. The mean time interval between the two examinations was less than one week. Exclusion criteria included image artifacts obscuring the spine, or any hardware or metal associated with the spine, as well as patients with spinal fractures, spinal tumors, endocrine diseases, rheumatic diseases, and infectious spondylitis, because these diseases are known to cause osteoporosis. Baseline clinic data, including the values of T11-L2 of fat-water images, age, gender, body mass index (BMI) derived from medical records were also recorded. The final cohort consisted of 95 patients(33 men, 62 women; mean age, 61.69 99.30 years).

\subsection{DXA Examination and Diagnostic Criteria of Osteoporosis}

DXA of the lumbar spine and proximal femora was performed for BMD assessment by using standard techniques according to manufacturer and WHO guidelines Hologicdiscovery dual-energy X-ray bone densitometer (Hologic Inc., Bedford, MA) [6]. According to WHO guidelines, a DXA-derived T-score less than -1.0 indicates an abnormally low BMD, which is further categorized into osteopenia (T-score between -1.0 and -2.5) and osteoporosis (T-score of -2.5 or below), and when the T-score is greater than -1.0 is normal. In our study, the patients were divided into two groups according to T-score, normal $\mathrm{BMD}(\mathrm{T} \geq-1)$ and abnormally low $\mathrm{BMD}(\mathrm{T}<-1)$.

\subsection{Spectral CT Examination}

All patients underwent routine thoracic or abdominal spectral CT with a scanner (Revolution CT; GE Healthcare, Wauwatosa, Wis) and a single-tube, fast dual-tube potential ( $80 \mathrm{kVp}$ and $140 \mathrm{kVp}$ ) switching scan technique. Imaging parameters were as follows: tube voltage $=80 / 140 \mathrm{KV}$; itch $=0.984$; rotation time $=0.8 \mathrm{~s} / \mathrm{r}$; slice thickness $=1.25 \mathrm{~mm}$, slice interval $=5 \mathrm{~mm}$, adaptive statistical iterative reconstruction $\mathrm{V}($ Asir-V) $=40 \%$.

\subsection{Image Segmentation and Feature Extraction}

The material decomposition images with fat-water as base materials obtained from the spectral CT imaging were transferred to ITK-SNAP software (Version 3.6.0, www.itksnap.org) for segmentation. An ovoid region of interest (ROI) of T11-L2 was manually drawn on the sagittal images with the same size, typically selecting a representative trabecular level centered between this inferior and the superior endplate(Figure1). Care was taken to avoid the posterior venous plexus, focal heterogeneity, cortical bone or any imaging-related artifacts. To assess the segmentation availability, the image segmentation was examined by another radiologist with 10 years of experience in CT interpretation. If the ROI was questioned, it would be re-segmented after the two agree.

AK software (AnalysisKit, version 3.2.0, GE Healthcare, China) backend with pyradiomics (version 3.0.1, https://pyradiomics.readthedocs.io/en/latest/) was used to extract the radiomics features. Before feature extraction, the images were preprocessed with 3 steps, resampling the voxel size into $1 * 1 * 1 \mathrm{~mm}^{\wedge} 3$, discretizing the gray values 
using 25 bin width, normalizing the gray value using $\mu \pm 3 \sigma$ method. After that, 6 classes of features were extracted, first-order features, gray level co-occurrence matrix features, gray level run length matrix features, gray level size zone matrix features, neighboring gray tone difference matrix features and gray level dependence matrix features, based on the original image, wavelet-transformed images and Laplacian of gaussian filtered images with sigma 2, 3. Totally, 828 features were extracted and used in the following analysis.

\subsection{Data preprocessing}

The dataset was randomly assigned in a 7:3 ratio to either the training cohort or test cohort. All cases in the training cohort were used to train the predictive model, while cases in the test cohorts were used to independently evaluate the model's performance.

Before analyses, variables with zero variance were excluded from analyses. Then, the missing values and outlier values were replaced by the median. Finally, the data were standardized using z-score method.

\subsection{Development of Radiomics Signature}

We used two feature selection methods, the minimum-Redundancy Maximum-Relevancy (mRMR) and the least absolute shrinkage and selection operator (LASSO) to select the feature. At first, mRMR was performed to eliminate the redundant and irrelevant features, 30 features were retained. Then LASSO was conducted to choose the optimized subset of features to construct the final model. Radscore was calculated by summing the selected features weighted by their coefficients.

\subsection{Development of Radiomics Nomogram and Assessment the Performance of Different Models}

The clinical variables included the values of T11-L2 of fat-water images, age, gender and BMI. We used the univariate logistic regression analyses to filter these variables and select the significant risk factors with $P<0.05$, subsequently, a backward step-wised multivariable logistic regression analysis with Akaike An Information (AIC) as criterion was performed to construct the clinical model. Meanwhile, a radiomics nomogram combining the final radiomics signature and independent clinical risk factors were built. Here, we used DeLong's test to compare whether the ROC curves were different between nomogram and clinical model. The calibration of the nomogram was evaluated with a calibration curve analysis and the Hosmer-Lemeshow test. Finally, we used decision curve to evaluate the clinical utility of the model.

\subsection{Statistical analysis}

All statistical analyses for the present study were performed with R(version 4.0.2, www.r-project.org). The mRMR and LASSO were used to select the features and were performed using 'mRMRe' and 'glmnet' packages, respectively. The LASSO includes choosing the regular parameter $\lambda$, determining the number of the feature. Wilcoxon test was applied to compared the radscores from abnormal low BMD group and normal BMD group on training cohort and test cohort respectively. Receiver operating characteristic(ROC) curve analysis was performed to evaluate the performance of the model, and area under curve (AUC) were calculated. Besides, DeLong's test was used to compare whether the ROC curves were different between nomogram and clinical model. A two-tailed p-value $<0.05$ indicated statistical significance.

\section{Results}

\subsection{Patients characteristics}


A total of 95 patients were included in our study. There were 67 and 28 patients in the training and test cohort respectively. The detailed patient characteristics in the two cohorts are displayed in Table 1. In each cohort, there was no significant differences in gender and age, but differences of the values of fat-water imaging sets were both detected in the two cohorts between the normal BMD patents and abnormally low BMD patents $(P=0.000)$. BMI was not statistically significant in the test cohort, however, it was statistically significant in the training cohort.

\section{Table 1}

Characteristics of patients in the training and test cohorts
Characteristics
Training cohort $(n=67)$
Test cohort(n=28)

\begin{tabular}{|c|c|c|c|c|c|c|}
\hline & Normal & $\begin{array}{l}\text { Abnormally } \\
\text { low BMD }\end{array}$ & $\begin{array}{l}P \text { - } \\
\text { values }\end{array}$ & Normal & $\begin{array}{l}\text { Abnormally } \\
\text { low BMD }\end{array}$ & $\begin{array}{l}P \text { - } \\
\text { values }\end{array}$ \\
\hline & BMD & & & BMD & & \\
\hline Number & 21 & 46 & & 9 & 19 & \\
\hline
\end{tabular}

Gender(\%)

Male

10(47.6)

12(26.1)

4(44.4)

7(36.8)

Female
11(52.4)
34(73.9)
$0.144 \quad 5(55.6)$
12(63.2)
1.000

Age, y,

$61.8 \pm 9.4$

$61.6 \pm 9.0$

0.933

$57.9 \pm 10.8$

$63.6 \pm 9.2$

0.1479

mean \pm SD

BMI, mean \pm SD

$25.8 \pm 2.3$

$23.9 \pm 3.5$

$0.021 * 26 \pm 2.9$

$24.4 \pm 2.6$

0.1467

Values of fat-water

imaging, mean \pm SD

$$
-3906.4 \pm 763.8 \quad-2590.3 \pm 718.4 \quad 0.000 * \quad-4491.3 \pm 1184.3 \quad-2581.2 \pm 603.3 \quad 0.000 *
$$

* $P$ value $<0.05$, two-sample t-test for continues variables; $c^{2}$ test and Fisher's exact test for categorized variables. BMD: bone mineral density; BMI: body mass index; SD: standard deviation.

\subsection{Feature selection}

828 features were finally extracted from one image. Due to none of features was 0 variance, 0 feature was removed. So that, 828 features were all imported into mRMR feature selection, which was performed to eliminate the redundant 
and irrelevant features, 30 features were retained. Then LASSO was conducted to choose the optimized subset of features to construct the final model(Figure 2). Finally, 25 features were removed and five features were selected as the most predictive subset of feature, which and their corresponding coefficients were shown in Figure 3.

\subsection{Radiomics signature construction}

Radscore was calculated by summing the selected five features weighted by their coefficients. The final formula of radscore is: Radscore $=-0.136{ }^{*}$ wavelet_LLH_glszm_ZonePercentage-0.756*original_firstorder_90Percentile$0.185 *$ wavelet_HLH_firstorder_Energy+0.641*wavelet_HLH_firstorder_Minimum0.109*original_glszm_SizeZoneNonUniformity +1.022 .

And we compared the radscores from normal BMD and abnormally low BMD of the fat-water imaging on training and test cohort respectively. Boxplots show the spectral CT radiomics signatures in abnormally low BMD patients were much higher than the normal BMD group in both the training and test cohort(Figuer 4). The AUC values of the radiomics model in the two cohorts were 0.95 (95\% Confidence interval [Cl]: 0.89-1.00, P凶0.001), 0.97 (95\% Cl: 0.91$1.00, P \otimes 0.001)$ respectively, indicating that radiomics features could effectively distinguish abnormally low BMD from normal BMD.

\subsection{Nomogram build}

By incorporating the values of fat-water imaging and radscores, a radiomics nomogram was developed in the training cohort (Figure 5) using the following formula: Nomoscore=(Intercept)*5.693+FatWater*0.0019+Radscore*1.855.

The ROC of radiomics nomogram and clinical factors model are exhibited in Figure 6 . Based on the radiomics nomogram and clinics model , the AUC values for training cohort were 0.96(95\% Cl: 0.91-1.00), 0.89(95\% Cl: 0.81-0.97), respectively; and the AUC values for test cohort were 0.98(95\% Cl: 0.93-1.00), 0.95(95\% Cl: 0.87-1.00), respectively. Based on the Youden Index, the diagnostic performance of every model is demonstrated in Table 2 . The radiomics nomogram showed better predictive efficacy than the clinics model both in the training cohort and test cohort, with the accuracy, sensitivity and specificity were $82.53 \%, 91.30 \%, 95.23 \%$ and $92.85 \%, 94.73 \%, 88.88 \%$ respectively. The cutoff of radiomics signature model is 0.786 , the cutoff of clinics model is 1.295 , the cutoff of radiomics nomogram model is 0.880 .

Table 2

Diagnostic efficiency of different models in the training and test cohorts 


\begin{tabular}{|c|c|c|c|c|c|c|}
\hline \multirow[t]{2}{*}{ Model } & \multirow[t]{2}{*}{ Accuracy(\%) } & Accuracy & Accuracy & \multirow[t]{2}{*}{ Sensitivity(\%) } & \multirow[t]{2}{*}{ Specificity(\%) } & AUC(95\%Cl) \\
\hline & & Lower(\%) & Upper(\%) & & & \\
\hline
\end{tabular}

Radiomics

\begin{tabular}{|c|c|c|c|c|c|c|c|}
\hline & Training & 91.01 & 81.52 & 96.64 & 89.13 & 95.23 & $\begin{array}{l}0.95(0.89- \\
1.00)\end{array}$ \\
\hline & Test & 82.14 & 63.10 & 93.93 & 78.94 & 88.88 & $\begin{array}{l}0.97(0.91- \\
1.00)\end{array}$ \\
\hline \multicolumn{8}{|l|}{ Clinics } \\
\hline & Training & 77.61 & 65.77 & 86.89 & 71.73 & 90.47 & $\begin{array}{l}0.89(0.81- \\
0.97)\end{array}$ \\
\hline & Test & 78.57 & 59.04 & 91.70 & 73.68 & 88.88 & $\begin{array}{l}0.95(0.87- \\
1.00)\end{array}$ \\
\hline \multicolumn{8}{|l|}{ Nomogram } \\
\hline & Training & 82.53 & 83.43 & 97.53 & 91.30 & 95.23 & $\begin{array}{l}0.96(0.91- \\
1.00)\end{array}$ \\
\hline & Test & 92.85 & 76.49 & 99.12 & 94.73 & 88.88 & $\begin{array}{l}0.98(0.87- \\
1.00)\end{array}$ \\
\hline
\end{tabular}

AUC:area under the curve; Cl: confidence intervals

The calibration curve of the radiomics nomogram demonstrated good agreement between the predicted and expected probabilities for normal BMD and abnormally low BMD in training cohort, and $P$ values of Hosmer-Lemeshow test were larger than 0.05 in both training and test cohorts. Finally, we used decision curve to evaluate the clinical usefulness of the model (Figure 7). It showed that the radiomics nomogram had a higher benefit in differentiating abnormally low BMD from normal BMD than the clinical factor model.

\section{Discussion}

In the present study, we developed and validated a radiomics nomogram that incorporated one clinical factor and five radiomics features derived from fat-water imaging based spectral CT, which can help clinician and radiologists to identify abnormally low BMD from normal BMD well. To the best of our knowledge, this is few radiomics model developed to diagnose osteoporosis combined with fat-water imaging based spectral CT.

Osteoporosis is characterized by bone loss and increased susceptibility to fragility fractures [15]. Osteoporosis are associated with lower osteogenesis and greater adipogenesis, and the both arise from a common mesenchymal stem cell within bone marrow [16,17]. The trabecular bone is the most metabolically active part in vertebrae [18]. Xiaojuan Li et al. found that the average fat content was significantly elevated of vertebral body in patients with osteoporosis/osteopenia compared with controls in 51 postmenopausal females by using magnetic resonance spectroscopy (MRS)[19]. Because DXA measures areal BMD, it can not differentiate between cortical and trabecular bone[20,21], it doesn't reflect fat content exactly as well.

Dual-energy CT measurements were used to derive basis material composition representation of the constituents of a measured volume. In a study by Bredella MA et al. [17], the L2 vertebra was scanned with dual-energy CT by using a dual source and multidetectors row CT scanner. They found excellent agreement between dual-energy CT and ${ }^{1} \mathrm{H}$ MRS in the assessment of marrow adipose tissue (MAT) content of L2 vertebra. Different from their study, the dual- 
energy CT used in our study was based on a single tube fast switching between low-energy and high-energy within a rotation, which was called spectral CT. In our study, the values from spectral CT fat-water MD imaging of T11-L2 were measured, and there were significant differences between normal BMD and abnormal low BMD. This result showed that the values of fat-water MD can reflect the change of marrow adipose tissue of osteoporosis. However, to the best of our knowledge, there are few studies using fat-water MD images of spectral CT as an evaluation method of osteoporosis.

Radiomics is a promising technique using computerized quantitative imaging analysis to extract a large number of image-related features to assist in diagnosing diseases, which has attracted increasing attention in recent years $[22,23]$. In the present study, LASSO method was adopted to reduce the regression coefficient to construct the radiomics signature, which has already been used for the prediction of bone metastasis in prostate cancer [24] and colorectal cancer [25] patients. Finally, radscore was calculated by summing the selected five features weighted by their coefficients. The radiomics feature model based on fat-water imaging of spectral CT showed sufficient discrimination in the training cohort (AUC $=0.95)$ and good predictive performance in the test cohort $(A U C=0.97)$.

In the present study, five radiomics features consisted of the optimal feature subset. In these 5 features, 3 were the firstorder features and 2 were GLSZM features. And original_firstorder_90Percentile and wavelet_HLH_firstorder_Minimum contribute more to the radiomics model for they have larger coefficients. That meant that the grey value distribution within the ROI between the normal and low BMD were different. Besides, GLSZM zone percentage and Size Zone Nonuniformity also had contributions to radiomics model, and their coefficients were negative, which meant that the normal BMD had fine texture and more homogeneity.

Gender and age were not statistically significant in the training and test cohort. Furthermore, these findings suggested that there were no differences of gender and age between abnormal and normal BMD. However, BMI was statistically significant in the test cohort only.

In addition to radiomics and clinics analysis, we also developed and evaluated the radiomics nomogram. The AUC, sensitivity, specificity, positive and negative predictive value of radiomics nomogram achieved more satisfactory than radiomics and clinics model both in training and test cohort. Our finding showed that the radiomics nomogram not only had higher sensitivity and specificity than the radiomics signature and the clinical model, but also had higher positive and negative predictive value both in training and test cohort. These findings suggested that the radiomics nomogram based on the combined model had a higher benefit in differentiating abnormally low BMD from normal BMD. Moreover, the decision curve showed that employing the nomogram could obtain more net benefits than the clinical model alone. Thus, the use of the developed nomogram may be a promising method in assisting radiologists in differentiating abnormally low BMD from normal BMD.

The limitations of our study should be acknowledged. Firstly, it was a retrospective study performed in a single institution. Secondly, the number of patients enrolled in our study was relatively small. Additionally, there were a few clinics features included in present study, only age, gender, BMI and the values of fat-water imaging. In the future, the limitations need to be further improved in the following investigations.

\section{Conclusions}

In conclusion, the present study developed a radiomics nomogram that incorporated clinical factor and radiomics features derived from fat-water imaging based spectral CT, which may serve as an efficient tool to help clinician and radiologists to identify abnormally low BMD from normal BMD well. 


\section{Abbreviations}

AIC Akaike an information

AUC Area under the curve

BMD Bone mineral density

BMI Body mass index

Cl Confidence interval

CT Computed tomography

DXA Dual x-ray absorptiometry

LASSO Least absolute shrinkage and selection operator

MAT Marrow adipose tissue

MD Material decomposition

mRMR Minimum-Redundancy Maximum-Relevancy

PACS Picture archiving and communication systems

ROC Receiver-Operating-Characteristic

\section{Declarations}

\section{Ethics Approval and Consent to Participate}

The institutional review board of The Second Affiliated Hospital of Shandong First Medical University approved this retrospective study. All methods were carried out in accordance with relevant guidelines and regulations. The need for obtaining signed informed consent was waived for retrospective analysis.

\section{Consent for publish}

Not applicable.

\section{Availability of data and materials}

The datasets used and/or analyzed during the current study are available from the corresponding author on reasonable request.

\section{Competing interests}

The authors declare that they have no competing interests. 


\section{Funding}

This study was funded by Academic Promotion Programme of Shandong First Medical University (No.2019QL017).

\section{Authors' Contributions}

QQY, MKL, KMY, YX, XQQ, XZZ, CQL,SFD and JQ designed the study. JQQ and SFD performed the data analysis. MKL, KMY and $Y X$ contributed to the data interpretation. XQQ, XZZ, CQL and JQ supervised the project. QQY wrote the manuscript and MKL, KMY, YX, XQQ, XZZ, CQL,SFD and JQ made contributions to its final form. All authors have read and approved the manuscript in this form.

\section{Acknowledgements}

We apologize to those authors we have not been able to cite due to space constraints.

\section{Authors' Information}

a Department of Radiology, The Second Affiliated Hospital of Shandong First Medical University,Taian,Shandong, 271000 ,China.

${ }^{b}$ GE Healthcare, Pudong new town, No1, Huatuo road, Shanghai 210000, China.

\section{References}

1. Cheng X, Yuan H, Cheng J, et al(2020). Chinese expert consensus on the diagnosis of osteoporosis by imaging and bone mineral density. Quant Imaging Med Surg. 10(10):2066-2077.

2. van Hamersvelt RW, Schilham AMR, Engelke K, et al(2017). Accuracy of bone mineral density quantification using dual-layer spectral detector CT: a phantom study. Eur Radiol. 27(10):4351-4359.

3. Hofbauer LC and Rachner TD(2015). More DATA to guide sequential osteoporosis therapy. Lancet. 386(9999):1116-1118.

4. Paola P, Daniela RM, Francesco C, et al(2016). Major osteoporotic fragility fractures: risk factor updates and societal impact. World J Orthop.7(3):171-181.

5. Jang S, Graffy PM, Ziemlewicz TJ, et al(2019). Opportunistic Osteoporosis Screening at Routine Abdominal and Thoracic CT: Normative L1 Trabecular Attenuation Values in More than 20000 Adults. Radiology. 291(2):360367.

6. Cheng X, Yuan H, Cheng J, et al(2020). Chinese expert consensus on the diagnosis of osteoporosis by imaging and bone mineral density. Quant Imaging Med Surg 10(10):2066-2077.

7. Fischer MA, Gnannt R, Raptis D, et al(2011). Quantifification of liver fat in the presence of iron and iodine: an exvivo dual-energy CT study. Invest Radiol. 46(6):351-358.

8. Ma Q, Yang Z, Han X, et al (2017). Influence of Parathyroidectomy on Bone Calcium Concentration: Evaluation with Spectral CT in Patients with Secondary Hyperparathyroidism Undergoing Hemodialysis-A Prospective Feasibility Study. Radiology. 284(1):143-152. 
9. Lv P, Lin XZ, Li J, et al(2011). Differentiation of small hepatic hemangioma from small hepatocellular carcinoma: recently introduced spectral CT method. Radiology 259(3):720-729.

10. Wu HZ, Zhang XF, Han SM, et al(2020). Correlation of bone mineral density with MRI T2* values in quantitative analysis of lumbar osteoporosis. Arch Osteoporos. 15(1):18.

11. Aerts HJ, Velazquez ER, Leijenaar RT, et al(2014). Decoding tumour phenotype by noninvasive imaging using a quantitative radiomics approach. Nat Commun. 5:4006.

12. Gillies RJ, Kinahan PE and Hricak H(2016). Radiomics: Images are more than pictures, they are data. Radiology. 278(2):563-577.

13. Gu D, Hu Y, Ding H, et al (2019). CT radiomics may predict the grade of pancreatic neuroendocrine tumors: a multicenter study. Eur Radiol. 29(12): 6880-6890.

14. Lambin P, Leijenaar RTH, Deist TM, et al(2017). Radiomics: the bridge between medical imaging and personalized medicine. Nat Rev Clin Oncol. 14(12):749-762.

15. Mao SS, Li D, Syed YS, et al(2017). Thoracic Quantitative Computed Tomography (QCT) Can Sensitively Monitor Bone Mineral Metabolism: Comparison of Thoracic QCT vs Lumbar QCT and Dual-energy X-ray Absorptiometry in Detection of Age-relative Change in Bone Mineral Density. Acad Radiol. 24(12):1582-1587.

16. Patsch JM, Li X, Baum T, et al(2017). Bone marrow fat composition as a novel imaging biomarker in postmenopausal women with prevalent fragility fractures. J Bone Miner Res. 28(8):1721-1728.

17. Bredella MA, Daley SM, Kalra MK, et al(2015). Marrow Adipose Tissue Quantification of the Lumbar Spine by Using Dual-Energy CT and Single-Voxel (1)H MR Spectroscopy: A Feasibility Study. Radiology. 277(1):230-235.

18. Engelke K, Adams JE, Armbrecht G, et al(2008). Clinical use of quantitative computed tomography and peripheral quantitative computed tomography in the management of osteoporosis in adults: the 2007 ISCD official positions. J Clin Densitom. 11(1):123-162.

19. Li X, Kuo D, Schafer AL, et al(2011). Quantification of vertebral bone marrow fat content using 3 Tesla MR spectroscopy: reproducibility, vertebral variation, and applications in osteoporosis. J Magn Reson Imaging. 33(4):974-979.

20. Link TM(2012). Osteoporosis imaging: state of the art and advanced imaging. Radiology. 263(1):3-17.

21. Krueger D, Fidler E, Libber J, et al(2014). Spine trabecular bone score subsequent to bone mineral density improves fracture discrimination in women. J Clin Densitom. 17(1):60-65.

22. Kang B, Sun C, Gu H, et al(2020). T1 Stage Clear Cell Renal Cell Carcinoma: A CT-Based Radiomics Nomogram to Estimate the Risk of Recurrence and Metastasis. Front Oncol. 10:579619.

23. Mao N, Dai Y, Lin F, et al(2020). Radiomics Nomogram of DCE-MRI for the Prediction of Axillary Lymph Node Metastasis in Breast Cancer. Front Oncol 10:541849.

24. Zhang W, Mao N, Wang Y, et al(2020). A Radiomics nomogram for predicting bone metastasis in newly diagnosed prostate cancer patients. Eur J Radiol. 128: 109020.

25. Huang YQ, Liang CH, He L, et al(2016). Development and Validation of a Radiomics Nomogram for Preoperative Prediction of Lymph Node Metastasis in Colorectal Cancer. J Clin Oncol.34(20): 2157-2164.

\section{Figures}




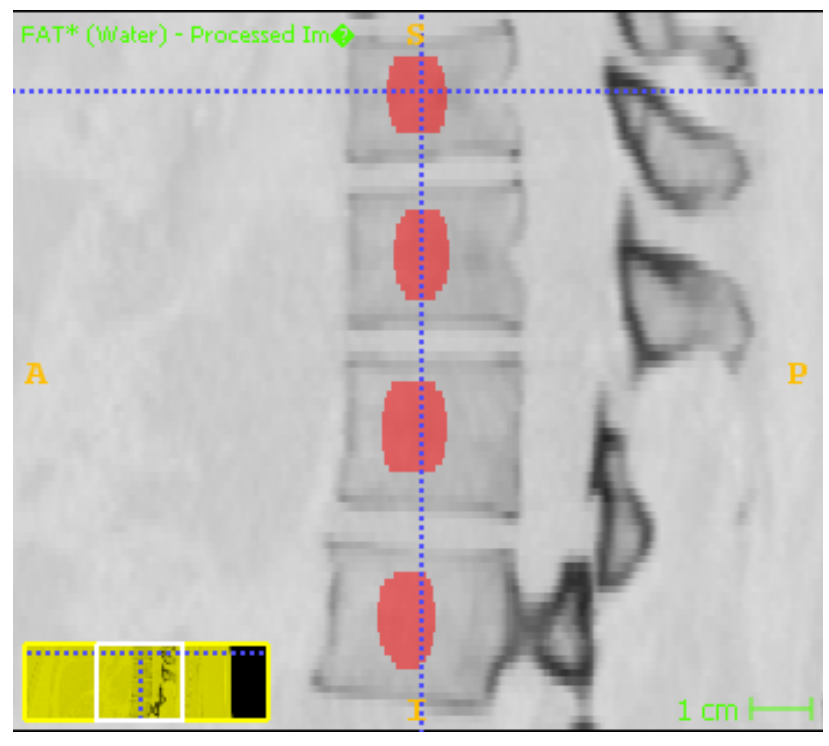

Figure 1

An ovoid region of interest (ROI) of T11-L2 was manually drawn on the sagittal images with the same size, typically selecting a representative trabecular level centered between this inferior and the superior endplate.
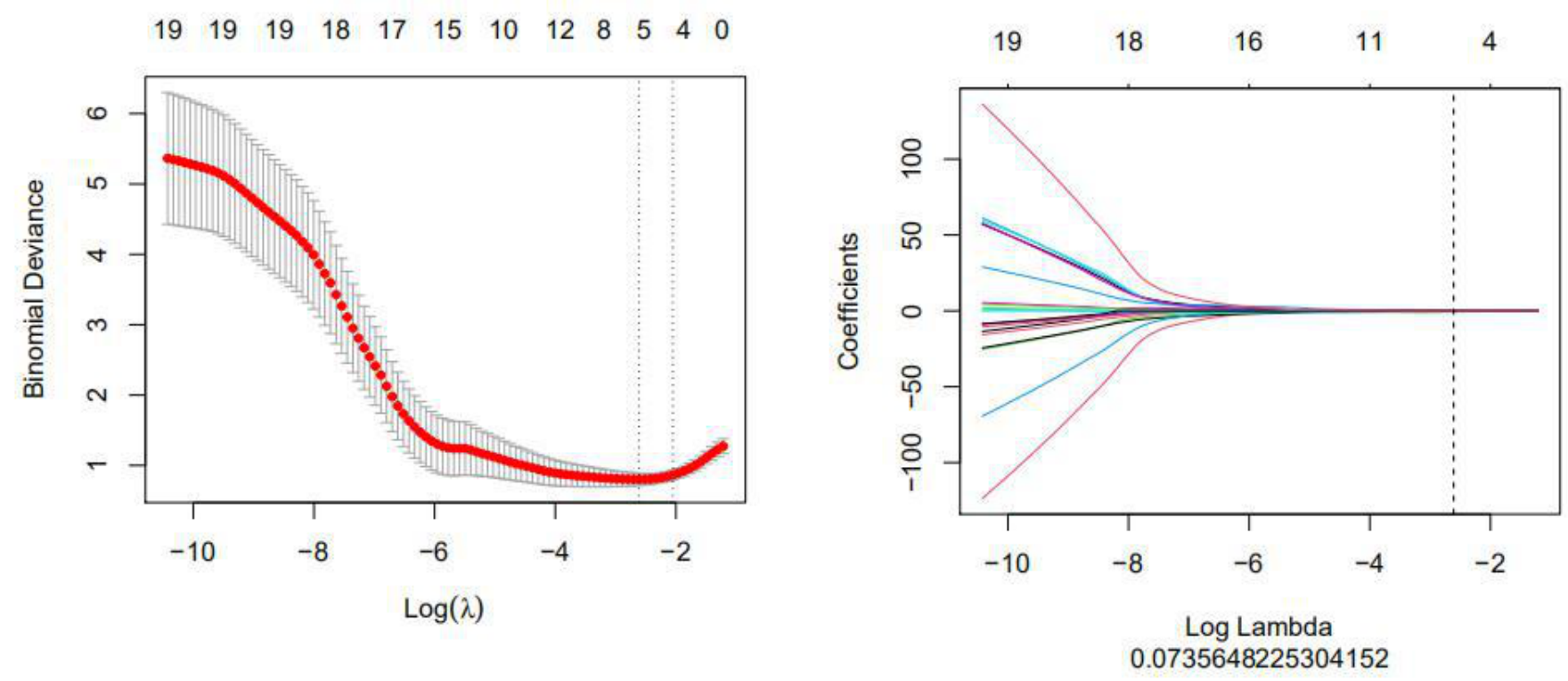

\section{Figure 2}

Selection of the regular parameter $(\lambda)$ in the LASSO model via 10 -fold cross validation based on minimum criteria. Binomial deviance was plotted as a function of $\log (\lambda)$. The optimal $\lambda$ value of 0.073 was selected. The dotted vertical line was drawn at the value selected using 10 -fold cross-validation, in which the selected $\lambda$ resulted in 5 non-zero coefficients. LASSO:the least absolute shrinkage and selection operator. 


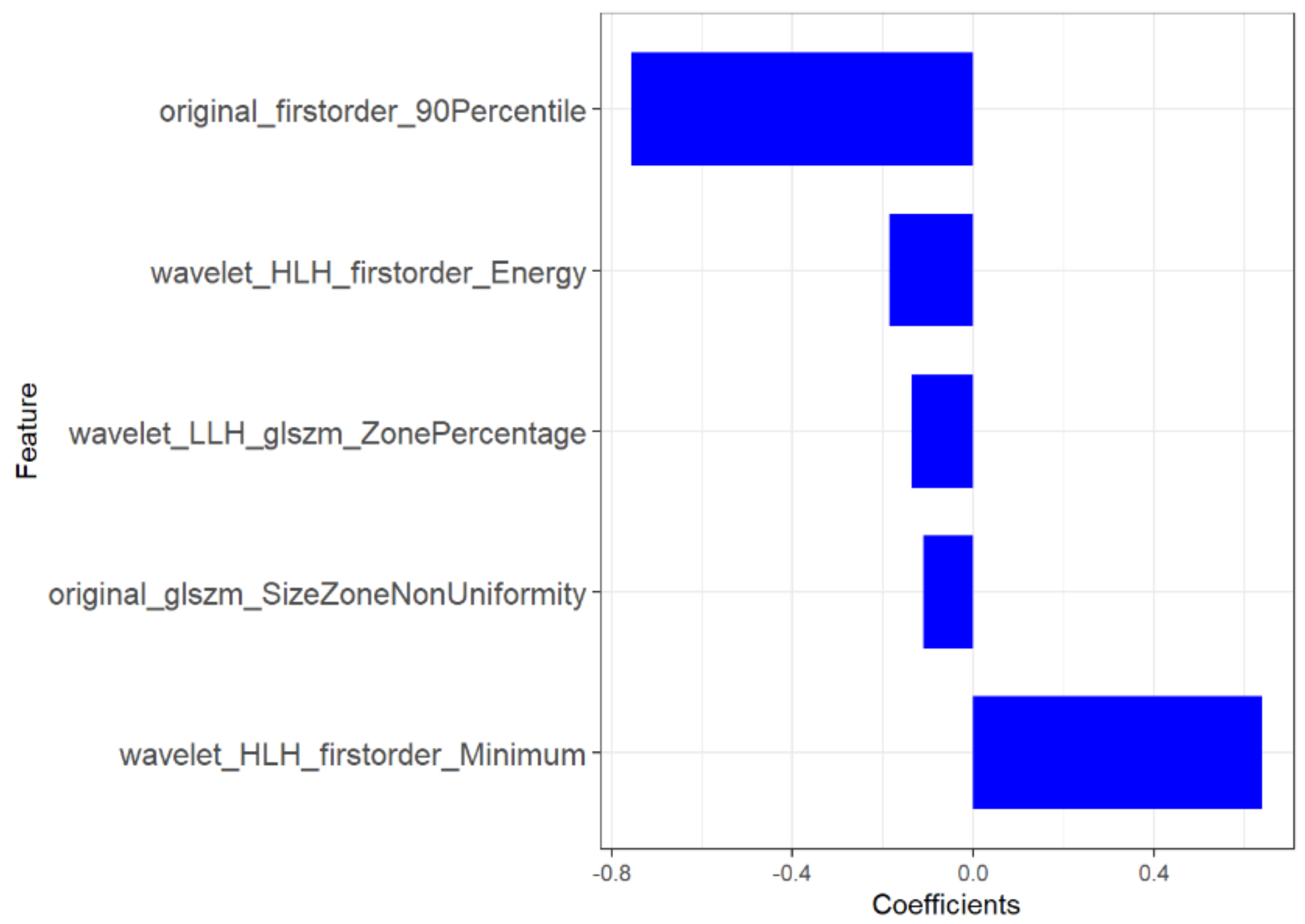

Figure 3

The final 5 features extracted from fat-water imaging of spectral CT and their coefficients after LASSO. LASSO:the least absolute shrinkage and selection operator. 

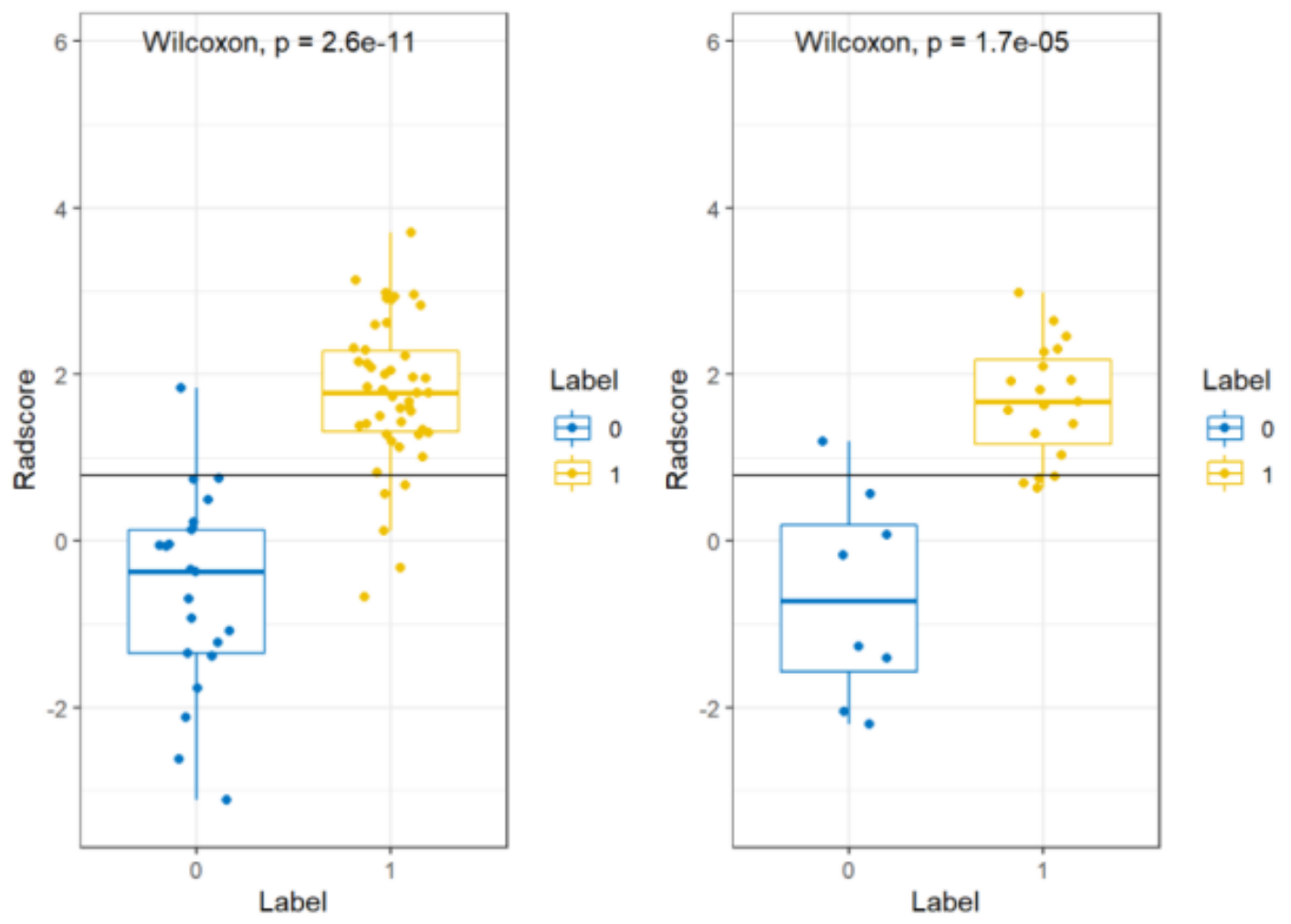

\section{Figure 4}

Boxplots show the spectral CT radiomics signatures in abnormal BMD patients were much higher than the normal BMD group in both the training (left) and test cohort (right). 0 means normal BMD group, 1 means abnormal BMD group.

Points

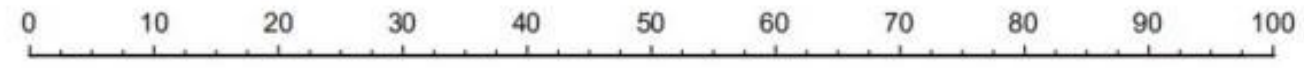

fat_water

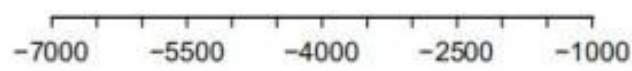

Radscore

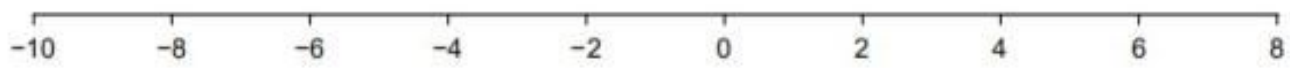

Total Points

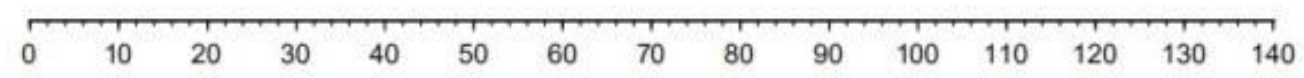

Risk

$$
0 . \overparen{10.40 .9}
$$

\section{Figure 5}

The radiomics nomogram, combining the values of fat-water imaging and radscores developed in the training cohort. 

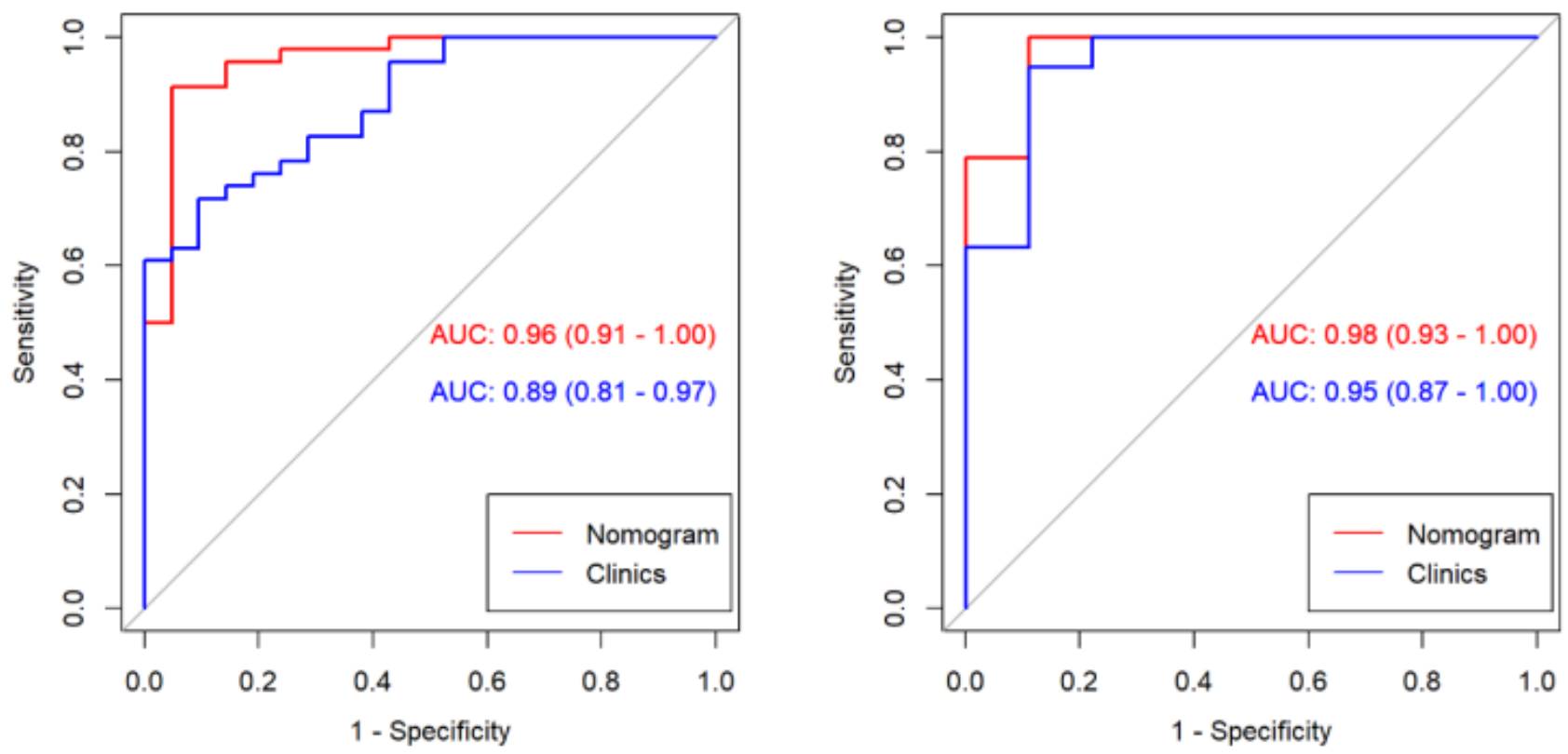

\section{Figure 6}

Comparison of ROC curves between the radiomics nomogram and clinical model for the prediction of BMD normal or abnormal in the training (left) and test (right) cohort.

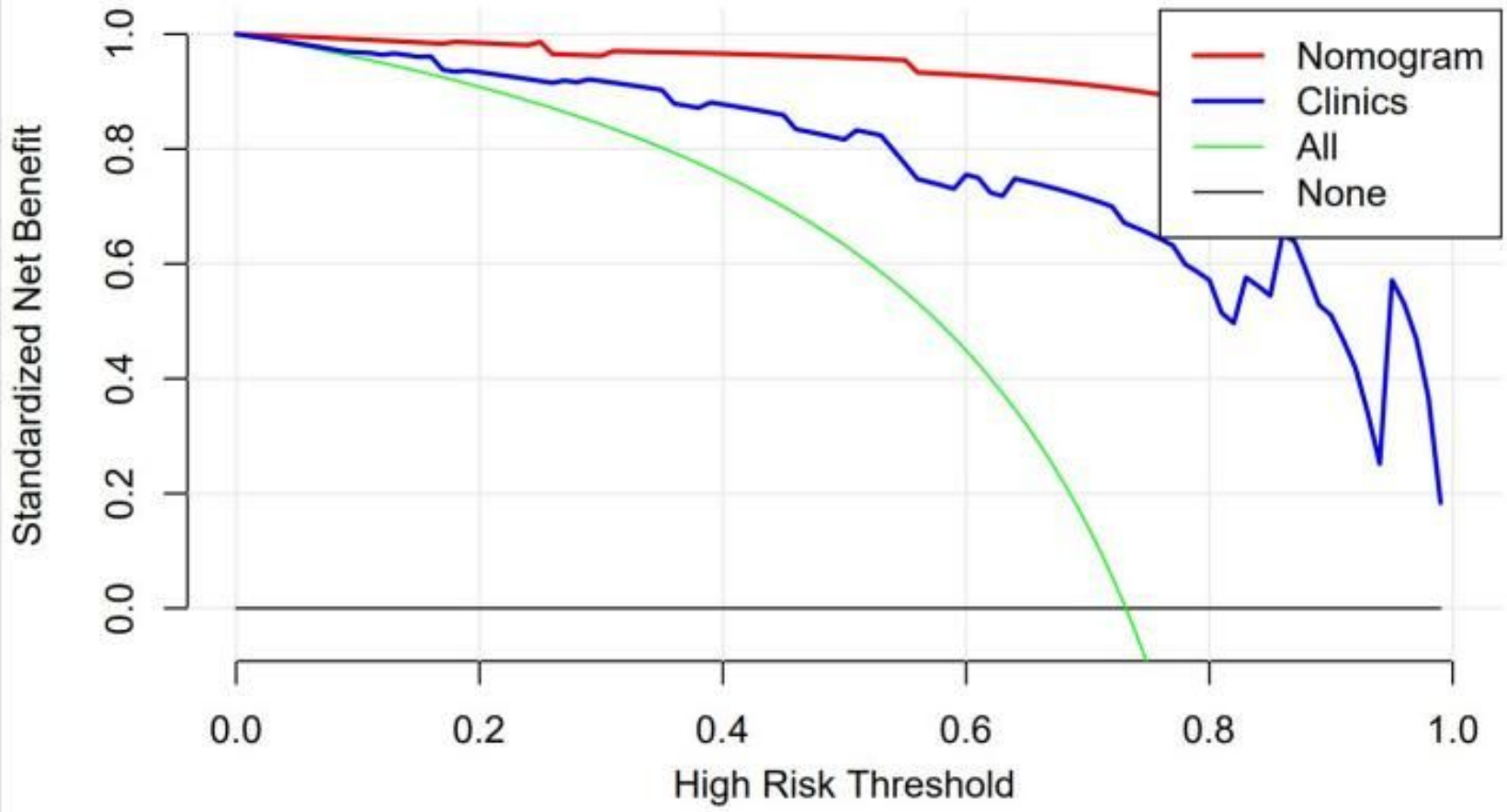

Figure 7

Decision curve analysis (DCA) for the radiomics nomogram. The DCA indicate that the application of radiomics nomogram to predict normal or abnomal BMD adds more benefit than clinical model. The red line and blue line represent the net benefit of the radiomics nomogram and the clinical model, respectively. The green line indicates the 
hypothesis that all patients had abnormal BMD. The black line represents the hypothesis that no patients had abnormal BMD. 